\title{
Fungal colonization of intragastric balloons
}

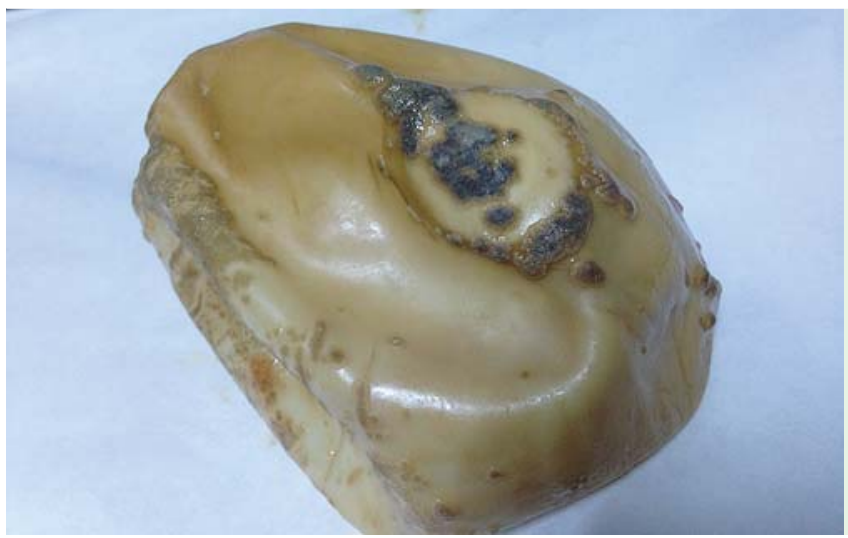

Fig. 1 Intragastric air balloon retrieved from a 35 -year-old woman (patient 1) 6 months after placement. The balloon was covered with multiple graywhite and brownishblack plaques.

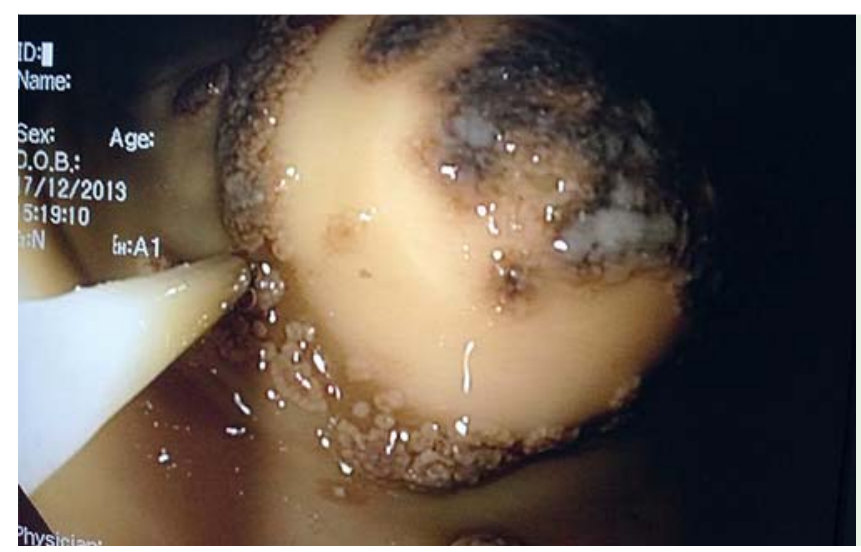

Fig. 2 Endoscopic view of the infected intragastric air balloon in a 45-year-old man (patient 2) 6 months after placement.

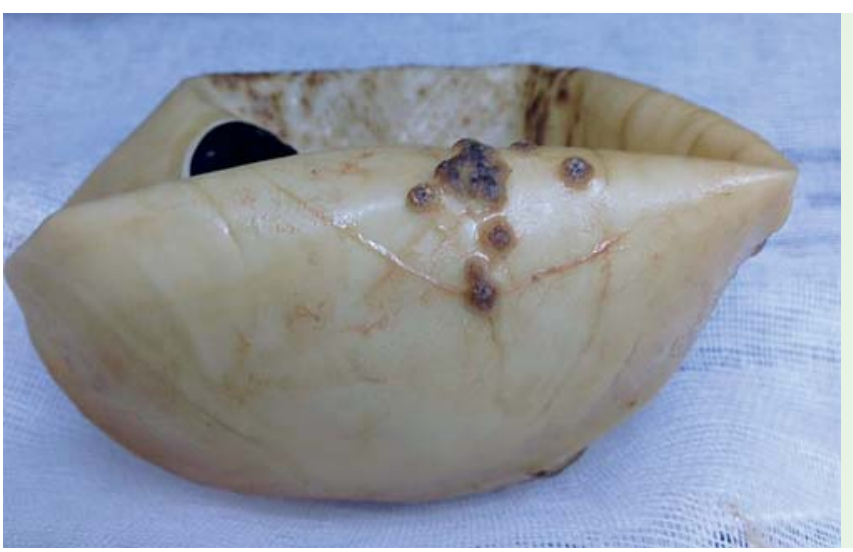

Fig. 3 Intragastric air balloon retrieved from patient 2, 6 months after placement. The balloon was deflated and covered in brownish-black plaques.

Intragastric balloon therapy is used for weight reduction as a short-term intervention in obese patients. The procedure is usually well tolerated, however, some complications including intolerance, vomiting, gastroesophageal reflux, gastric erosions and esophagitis, gastric ulcers, and spontaneous deflation of the balloon have been observed. In addition, gastric perforation, dilatation, and small-intestinal obstructions are occasionally reported $[1,2]$. Different types of intragastric balloon may be used for weight reduction [3]. Herein, we present three asymptomatic patients with intragastric balloons infected by Candida albicans yeast colonies.

In all three patients, a Heliosphere BAG intragastric air balloon (Helioscopie, Vienne, France) was inserted under propofol sedation for weight reduction. The balloons were removed after 6 months. We used a Heliosphere Newtech extraction kit (Helioscopie) to retrieve the bal-

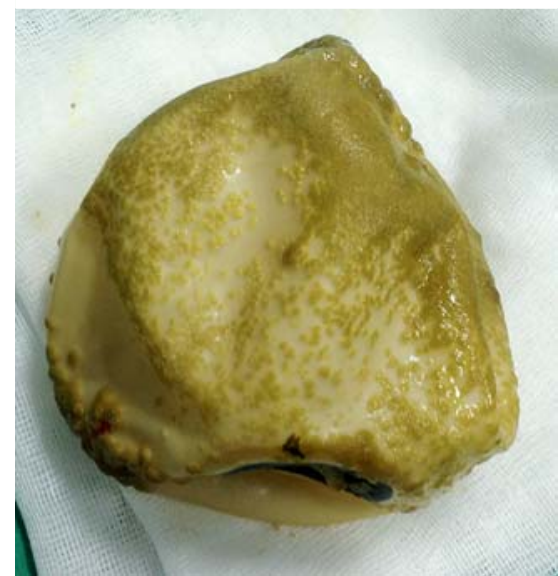

Fig. 4 Intragastric air balloon retrieved from a 45-year-old woman (patient 3) 6 months after placement. The balloon was covered with multiple necrotic, greenish-yellow plaques.

loons. The kit consists of a needle catheter to deflate and air aspirate the balloon, and a foreign body grasper to remove the balloon gently.

The first patient was a 35-year-old woman, $126 \mathrm{~kg}$ in weight and with body mass index (BMI) $45.2 \mathrm{~kg} / \mathrm{m}^{2}$. During retrieval of the balloon, endoscopic findings were unremarkable, but the surface of the balloon was covered with necrotic gray-white and brownish-black plaques ( Fig. 1). The second patient was a 45-year-old man. His weight was $127 \mathrm{~kg}$ and BMI was $43.9 \mathrm{~kg} / \mathrm{m}^{2}$. After 6 months, we observed, while retrieving the balloon, that it was deflated and covered in brownish-black plaques ( Fig. 2 and $\bullet$ Fig. 3 ). The third patient was a 45 -year-old woman, $130 \mathrm{~kg}$ in weight and BMI $48.3 \mathrm{~kg} / \mathrm{m}^{2}$. Upon removal, we observed multiple yellowishgreen plaques on the surface of the balloon ( $\bullet$ Fig. 4). The patients were all asymptomatic. There were no remarkable findings in the gastric mucosa of the latter two patients. Microbiological examination of these plaques revealed Candida albicans colonization ( $\bullet$ Fig. 5 and $\bullet$ Fig. 6 ).

Fungal and bacterial colonization can occur on the surface of intragastric balloons. Various predisposing factors such as gastric stasis, antiacid drugs, and smoking may play a role in opportunistic infections in patients with intragastric balloons [4]. Spontaneous deflation of the balloon may be a risk factor as in the second patient discussed above. This should be taken 


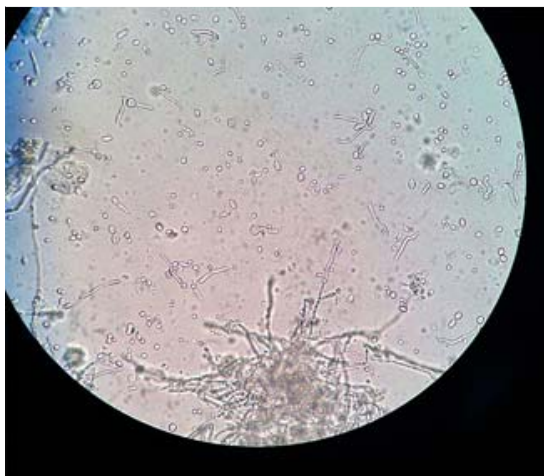

Fig. 5 The infected layer was wiped with a sterile swab and the material examined microscopically $(\times 40)$ in a wet mount preparation. The material was inoculated onto blood agar, eosin methylene blue agar, and Sabouraud dextrose agar. After incubation for 24 hours at $37^{\circ} \mathrm{C}$, small gray-white colonies grew on the blood agar. Candida albicans was identified with a BD Phoenix 100 instrument (Becton-Dickinson, Sparks, Maryland, United States).

into consideration, especially in immunosuppressed patients, and these patients should be monitored. If any asymptomatic balloon infection occurs, the patient should be treated, especially those with damaged gastrointestinal system mucosa.

Endoscopy_UCTN_Code_CPL_1AH_2AK

Competing interests: None

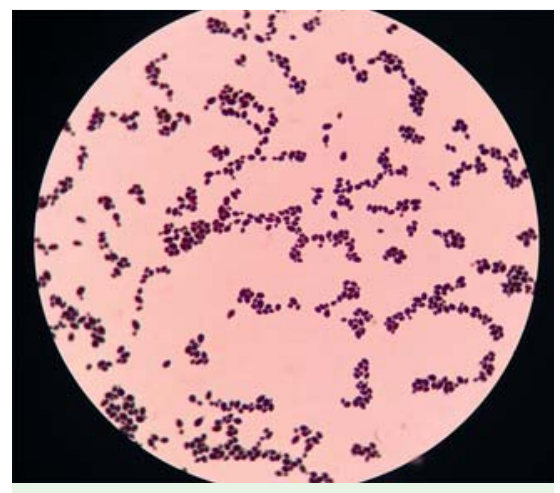

Fig. 6 Gram staining of the plaques revealed Candida species yeast colonies. Antimicrobial susceptibility testing was performed with a Fungitest Microplate (Bio-Rad, Mames-la-Coquette, France) and Candida albicans species were detected as susceptible to miconazole, ketoconazole, amphotericin B, fluconazole, flucytosine, and itraconazole.

\section{Zahide Şimşek ${ }^{1}$ Oğuz Alp Gürbüz², Şahin Çoban ${ }^{1}$}

${ }^{1}$ Department of Gastroenterology, Diskapi Yıldırım Beyazıt Education and Research Hospital, Ankara, Turkey

2 Department of Microbiology, Diskapi Yıldırım Beyazıt Education and Research Hospital, Ankara, Turkey

\section{References}

1 Gostout CJ, Rajan E. Endoscopic treatments for obesity: past, present and future. Gastroenterol Clin North Am 2005; 34: 143 - 150

2 Dumonceau JM. Evidence-based review of the Bioenterics intragastric balloon for weight loss. Obes Surg 2008; 18: $1611-$ 1617

3 Tsesmeli N, Coumaros D. Review of endoscopic devices for weight reduction: old and new balloons and implantable prostheses. Endoscopy 2009; 41: $1082-1089$

4 Coskun H, Bozkurt S. A case of asymptomatic fungal and bacterial colonization of an intragastric balloon. World J Gastroenterol 2009; 15: $5751-5753$

\section{Bibliography}

DOI http://dx.doi.org/

10.1055/s-0034-1390838

Endoscopy 2014; 46: E642-E643

(c) Georg Thieme Verlag KG

Stuttgart · New York

ISSN 0013-726X

\section{Corresponding author}

\section{Sahin Coban, MD}

Department of Gastroenterology Diskapi Yıldırım Beyazıt Education and Research Hospital

Ankara

Turkey

Fax: +90-312-3186690

scoban72@yahoo.com 